\title{
In vitro Toxicity Testing of Nanoparticles in 3D Cell Culture
}

\author{
Jungwoo Lee, G. Daniel Lilly, R. Christopher Doty, Paul Podsiadlo, and \\ Nicholas A. Kotov*
}

\section{Com} tissues that have extensive cell-cell and cell-matrix interactions, as well as markedly different diffusion/transport conditions. Hence, testing cytotoxicity in $2 D$ cultures may not accurately reflect the actual toxicity of nanoparticles (NPs) and other nanostructures in the body. To obtain more adequate and detailed information about NP-tissue interactions, we here introduce a 3D-spheroid-culture-based NP toxicology testing system. Hydrogel inverted colloidal crystal (ICC) scaffolds are used to create a physiologically relevant and standardized $3 D$ liver tissue spheroid model for in vitro assay application. Toxicity of CdTe and Au NPs are tested in both $2 D$ and $3 D$ spheroid cultures. The results reveal that $N P$ toxic effects are significantly reduced in the spheroid culture when compared to the $2 D$ culture data. Tissue-like morphology and phenotypic change are identified to be the major factors in diminishing toxicity. Acting as an intermediate stage bridging in vitro $2 D$ and in vivo, our in vitro $3 D$ cell-culture model would extend current cellular level cytotoxicity to the tissue level, thereby improving the predictive power of in vitro NP toxicology.

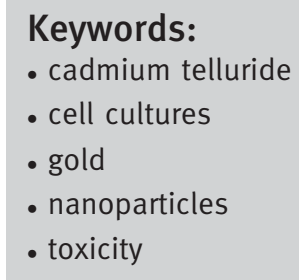

Keywords:

- cadmium telluride

- cell cultures

gold

- toxicity

\section{Introduction}

Virtually all cells in the body reside in a 3D environment, which is critical for their growth and metabolism. ${ }^{[1]}$ The phenotype and function(s) of individual cells are highly dependent on sophisticated interactions with 3D-organized extracellular matrix (ECM) proteins and neighboring cells. ${ }^{[2]}$

[*] Prof. N. A. Kotov, J. Lee, G. D. Lilly, P. Podsiadlo

Department of Biomedical Engineering, Chemical Engineering, Material Science and Engineering

University of Michigan

3074 H. H. Dow Building

2300 Hayward Street

Ann Arbor, MI 48109 (USA)

E-mail: kotov@umich.edu

Dr. R. C. Doty

Nico Technologies Co.

Ypsilanti, MI 48198 (USA)

․ Supporting Information is available on the WWW under http:// www.small-journal.com or from the author.

DOI: $10.1002 /$ smll.200801788
However, these cell-cell and cell-matrix interactions are considerably reduced in the case of $2 \mathrm{D}$ cell culture on a flat substrate, which in turn significantly limits their ability to recapitulate the appropriate level of in vivo cellular responses. ${ }^{[3]}$ Therefore, despite providing valuable and important information, tests based on in vitro 2D-cell-culture models do not accurately predict in vivo toxicity and other biological effects due to the absence of key physiological processes $^{[4]}$ such as transport of nanoparticles (NPs) through cell layers when they are brought in contact with the tissues. Also, essential effects of NPs (and other substances) are neglected with respect to cellular functions that are strongly dependent on 3D organization. For example, the enhanced specific protein secreting function of granular epithelial cells can only be observed when they form a 3D-organized acinus structure. ${ }^{[5]}$ As an additional piece of evidence substantiating the significance of expanding cell toxicity assays from $2 \mathrm{D}$ to $3 \mathrm{D}$ cultures, one also must mention that it has become increasingly apparent that there is a large discrepancy in toxicity results depending on whether in vitro 2D-cell-culture or animal models were used. ${ }^{[6]}$ For instance, recent studies on toxicity testing of quantom dots, ${ }^{[7]}$ magnetic NPs, ${ }^{[8]}$ carbon 
nanotubes, ${ }^{[9]}$ and fullerenes ${ }^{[10]}$ using in vitro $2 \mathrm{D}$ cell culture showed high cytotoxic effects. However, when they were tested in animal models, no adverse effects were observed. ${ }^{[1-14]}$

In vitro 3D-cell-culture models have been introduced to bridge the gap between in vitro $2 \mathrm{D}$-cell-culture and in vivo models. ${ }^{[15]}$ Multicellular spheroid culture is expected to be the most effective 3D-cell-culture model. ${ }^{[16,17]}$ Extensive cell-cell interactions in a 3D-assembled sphere-shaped cell colony promote the recovery of original tissue morphology and function. For example, histological and electron microscopic analysis of the structure of human tumors and their spheroid counterparts are nearly indistinguishable. ${ }^{[18]}$ Spheroid culture of hepatocytes has demonstrated excellent viability while preserving the liver's metabolic functions. ${ }^{[19]}$ For that reason, it is expected that toxicity testing based on physiologically relevant spheroid models can extend current cellular level cytotoxicity to the tissue level. ${ }^{[4,17]}$ Therefore, it can enhance the predictive power of current in vitro toxicity testing for estimating in vivo toxicity.

Methods for the toxicity testing of NPs are basically the same as the techniques used in modern drug development, however, the toxic mechanism of NPs can be more diverse than that of drug compounds. NPs can be indiscriminately transported into cells due to their comparable dimension to biological macromolecules. ${ }^{[20]}$ Fiber-shaped NPs such as nanowires, nanotubes, and nanofibers have a high probability for the penetration of cell membrane and tissue layers, as has been reported for asbestos. ${ }^{[21]}$ Decomposed NPs generate free radical species or toxic ions that can injure plasma membrane functions by reducing membrane integrity or impairing ion channel transport. ${ }^{[22]}$ Additionally, there are potentially more unrecognized harmful effects of NPs considering the great diversity of engineered NPs in chemical composition, size, shape, charge, coating, solubility, and so on. ${ }^{[2-25]}$ Equally, there might be some potentially unrecognized beneficial effects related to the same factors. Until now all of the in vitro NP toxicity testing has been performed using 2D cell cultures, ${ }^{[26]}$ and it will be very important to demonstrate a suitable 3D cell model for NPs and compare the results with 2D cell cultures.

In the present report, we introduce for the first time a 3Dspheroid-culture-based NP toxicity testing system. Our initial target is preparing 3D-liver-tissue-spheroid models with the human hepatocarcinoma (HepG2) cells since the liver is the major organ for NP accumulation. ${ }^{[27]}$ Inverted colloidal crystal (ICC) topology prepared from a transparent and cell repulsive polyacrylamide hydrogel was used as a 3D-cell-growth substrate. ${ }^{[28]}$ The ICC geometry induces spheroid formation of HepG2 cells, and optical observation is readily accessible through the transparent hydrogel matrix. Mature spheroids can be stably entrapped into ICC pores since their diameter became larger than channel dimension. Importantly, the stringently controlled topology of ICC scaffolds made of monodispersed micrometer-sized beads results in a narrow size distribution of spheroid diameters. As a result, we obtained homogeneous HepG2 spheroid arrays that permit the systematic and reproducible characterization of the effect of NPs on liver tissue. The toxic effects of CdTe and Au NPs were tested using a number of different approaches, including morphology, membrane integrity, metabolic activity, and cell death mechanism, and a comparison was made with conventional 2D-culture-based cytotoxicity. Our results clearly indicate the significance of the 3D-cell-culture model to in vitro testing of NP toxicity and the need of implementing standardized 3D in vivo models for NP research.

\section{Results and Discussion}

\subsection{Pocket ICC Scaffold Design}

Standardization of spheroid diameters and total cell numbers are critical issues to obtaining reproducible analytical results from 3D cultures. The biological activity of a spheroid is closely related to the size of their diameter. ${ }^{[29]}$ For instance, spheroids having excessively small diameters would not have the proper tissue level of physiological properties and would instead remain at the cellular level due to the lack of cell-cell interactions. Increasingly large diameters cause cells at the center of the spheroid to suffer from hypoxia and inadequate nutrient transport owing to the limited diffusion of oxygen and nutrients. ${ }^{[16]}$ As a result, a significant portion of cells ultimately undergo necrosis. Therefore, to achieve a homogeneous and meaningful level of biological properties, spheroids should have appropriate diameters with the narrowest size distribution possible. Also, total cell numbers need to be consistently regulated for the convincing quantification of intra- and extracellular proteins because these assay results are intrinsically dependent on the number of cells involved.

Our approach started with recently introduced polyacrylamide hydrogel ICC scaffolds. ${ }^{[28]}$ The primary structure of an ICC scaffold consists of highly organized and uniformly sized spherical pores. ${ }^{[30]}$ Polyacrylamide hydrogel is well-known for its biocompatibility, transparency, and nonfouling properties. Therefore, the ICC topology created with a cell-repulsive hydrogel matrix exhibits excellent physical and chemical properties for spheroid formation with a narrow size distribution while simultaneously retaining a high optical analytic capability. However, in the previous variants of ICC scaffold design, a number of cells were released right after seeding from the bottom of the scaffold due to the completely interconnected and open porous ICC structure, which caused difficulty in controlling the total cell number within the scaffold. Moreover, the released cells grew in a $2 \mathrm{D}$ environment on the bottom of the well-plate, hindering the reproducibility of the experiment and reducing the $3 \mathrm{D}$ culture effect (Figure 1A and Supporting Information Figures 1 and 2). To circumvent this issue, we modified the ICC scaffold design to have open pores only on the top side, which is used for cell seeding. Pores on the bottom and edge planes are enclosed by bulk hydrogel to reduce the chance for cell loss (Figure 1B). Although macroscale pores are sealed, sufficient oxygen and nutrient exchange is still maintained in the scaffold due to the presence of submicrometer-scale pores in the bulk hydrogel. Importantly, once individually seeded cells form multicellular spheroids, the spheroids can permanently remain within each pore because their diameters become larger than the channel dimension (Figure 1C). In this way, we can 
A

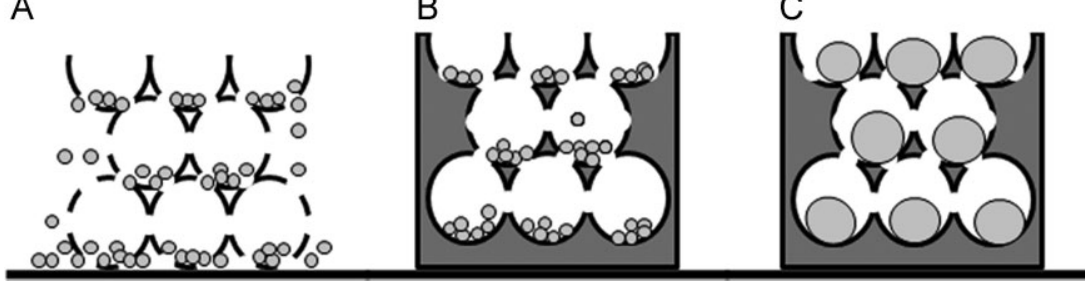

Figure 1. Schematic of cell seeding and spheroid growth in ICC scaffolds. A) Diagram of an open ICC scaffold that loses a significant number of cells right after seeding. B) A pocket ICC scaffold that considerably reduces cell loss through its closed bottom and edge plane pores. C) After 3-5 days of culture, single cells form spheroids whose diameters become larger than the channel size. As a result, the solid spheroid can stably remain in the pore. In a way, the ICC scaffold serves as a pocket for spheroids.

minimize cell loss during seeding and stably maintain total cell numbers, a distinct advantage for all assay applications.

\subsection{Liver Tissue Spheroid Preparation}

An absolute spheroid size suitable for toxicity testing and other assays has not been identified. Considering the previously reported data, ${ }^{[31,32]}$ we aimed to control spheroid diameters in this study at around $100 \mu \mathrm{m}$ so as not to induce hypoxic culture conditions while recovering tissue-level physiological properties. The spheroid diameters are dependent upon pore dimensions, which can be easily regulated by changing the size of the microspheres in the colloidal crystal template. Here, we used glass beads with a diameter of $156 \pm 8.4 \mu \mathrm{m}$ for preparing colloidal crystals. Final ICC scaffolds have $174.6 \pm 10-\mu \mathrm{m}$-diameter pores, approximately $10 \%-13 \%$ larger than the template bead sizes due to swelling of the hydrogel matrix, and $49.6 \pm 7-\mu \mathrm{m}$-diameter interconnected channels, approximately $30 \%$ of pore diameters (Figure 2A and B). The overall dimensions of a cylindricalshaped pocket ICC scaffold were $6.5 \mathrm{~mm}$ in diameter with a thickness of $1-1.5 \mathrm{~mm}$, including $0.5-0.8 \mathrm{~mm}$ of bulk hydrogel on the bottom side (Figure 2C).

Other important factors controlling spheroid diameter include cell seeding density and methods that determine the homogeneous distribution of the appropriate number of cells in each pore. In order to achieve uniform cell distribution and minimize cell loss during seeding, we inoculated a small volume of a dense cell suspension $\left(5 \times 10^{5}\right.$ cells per $\left.20-30 \mu \mathrm{L}\right)$ on top of a slightly dried hydrogel scaffold. The entire cell suspension solution readily permeates into the dehydrated hydrogel ICC scaffold, improving the quality and efficiency of cell seeding. After 3-5 days of culture, the individual cells formed solid spheroids with constant diameter. The measured spheroid diameters were on average $99.8 \pm 14 \mu \mathrm{m}$, which is approximately $56 \%$ of the pore diameter and $180 \%$ of the channel diameter (Figure 2D). Indeed, single spheroids could permanently reside in one pore due to the "ship-in-a-bottle" effect that significantly improved the quality of 3D-spheroidbased assay results by keeping the total amount of cells constant. It also exhibited other advantages, such as preventing spheroids from merging, because without physical barriers spheroids tend to aggregate, and keeping spheroids in the same position, which allows the tracing and 3D imaging of spheroids under optical or confocal microscopes.

\subsection{Morphological Evaluation of NP Toxicity in 3D Spheroids}

We tested two types of NPs: CdTe and $\mathrm{Au}$, which hold great potential for various biomedical applications. The exact mechanism of semiconductor NP (e.g., CdTe and CdSe)-induced toxicity is unclear, however, it has been identified that the most important aspect is the stability of NPs under physiological conditions, that is, intracellular and in vivo, as well as during synthesis and storage since they are susceptible to photolysis and oxidation. Released free cadmium and reactive oxygen species impair cell function and eventually kill the cells..$^{[7,33]}$ For example, Derfus et al. demonstrated that decreased photostability of CdSe NPs under exposure to ultraviolet light caused liberation of free $\mathrm{Cd}^{2+}$, which in turn enhanced cytotoxicity. ${ }^{[34]}$ Kirchner et al. reported that the core-shell structure of $\mathrm{CdSe} / \mathrm{ZnS}$ significantly reduced the cytotoxicity of CdSe NPs by protecting the core from oxidation and preventing it from leaching into the surrounding solution. ${ }^{[35]}$ The cytotoxicity of semiconductor NPs also differs depending on their size ${ }^{[11,33]}$ and stabilizer chemistry, and surface modification. ${ }^{[7,36]}$ On the other hand, Au NPs have excellent stability and major factors inducing toxic effect are shape, size, and stabilizer chemistry. ${ }^{[37,38]}$

In this study, we intentionally used unmodified L-cysteinestabilized CdTe NPs, which are unstable and quite toxic, in
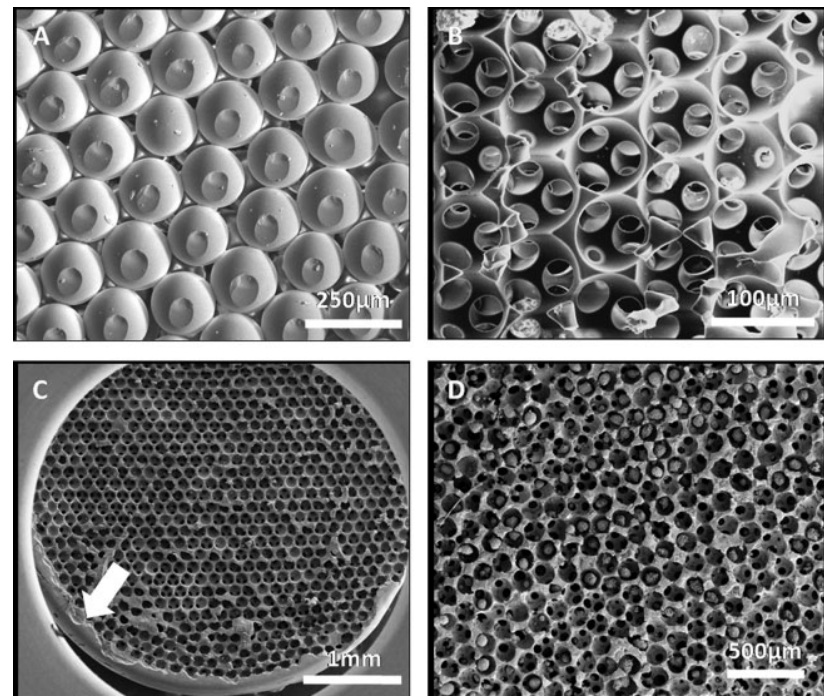

Figure 2. 3D liver tissue spheroids formation in hydrogel ICC scaffolds. SEM image of A) a colloidal crystal template prepared with $156 \pm 8.4-\mu \mathrm{m}$-diameter glass microspheres, B) ICC geometry created with polyacrylamide hydrogel, C) a pocket ICC scaffold (the arrow indicates a pocket wall), and D) a multicellular spheroid formation of HepG2 cells after 5 days of culture. The hydrogel scaffolds and spheroids were dehydrated with ethanol before imaging, causing shrinkage of both pore and spheroid dimensions. 

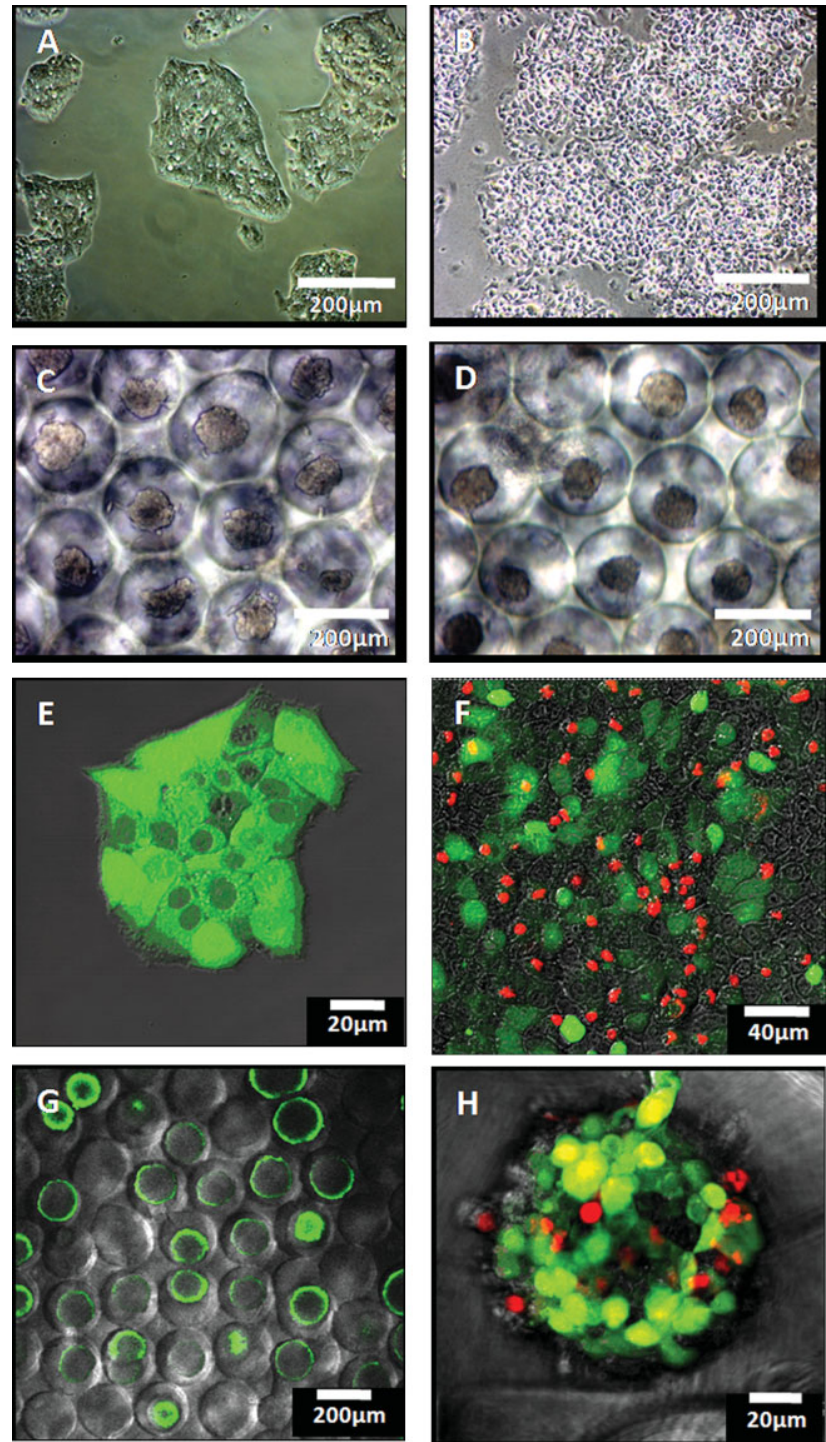

Figure 3. Comparison of $2 \mathrm{D}$ and $3 \mathrm{D}$ culture of HepG 2 cells after $12 \mathrm{~h}$ of CdTe NP exposure. A-D) Optical images of normal A) 2D and C) 3D spheroid cultures. After CdTe NP introduction, the 2D culture showed a dramatically different morphology (B), while it was hard to distinguish any change in the 3D culture under an optical microscope (D). E-H) Confocal images of live/dead-stained normal E) 2D and G) 3D spheroid cultures; live cells are green and dead cells are red. Most cells in both cultures showed excellent viability. F) Again after CdTe NP exposure, 2D culture revealed that a significant number of cells were dead. H) Although a few cells located on the surface of spheroids were dead, overall the number is much smaller than the $2 \mathrm{D}$ culture.

order to highlight the different cellular effects between 2D and 3D spheroid cultures. The CdTe NPs have a photoluminescence peak at $577 \mathrm{~nm}$ and an average diameter of $D=$ $2.9 \pm 1.0 \mathrm{~nm}$ (Supporting Information Figure 3). The CdTe $\mathrm{NP}$ concentration was constantly maintained at $10 \mu \mathrm{g} \mathrm{mL} \mathrm{m}^{-1}$ while varying the exposure time up to $24 \mathrm{~h}$. In the case of $\mathrm{Au}$ NPs, we tested citrated-stabilized $(D=3.5 \pm 0.7 \mathrm{~nm})$ and cetyltrimethylammonium bromide (CTAB)-stabilized $(D=5.5 \pm 0.6 \mathrm{~nm})$ spherical Au NPs. Their concentration and exposure time were maintained at $98.5 \mu \mathrm{g} \mathrm{mL}^{-1}$ of $\mathrm{Au}$ and $24 \mathrm{~h}$, respectively. All toxicity testing was performed with freshly synthesized NPs less than a week after preparation, but we observed increased toxic effects of CdTe NPs over time due to decomposition (Supporting Information Figure 4). In addition, to establish comparable testing conditions in both $2 \mathrm{D}$ and $3 \mathrm{D}$ cultures, we kept the same culture volume $(1 \mathrm{~mL})$ and a similar number of cells at the point of toxicity testing. Since the cell proliferation rate in spheroid culture is considerably slower than in $2 \mathrm{D}$ culture, five times fewer cells were seeded for $2 \mathrm{D}$ cultures $\left(1 \times 10^{5}\right.$ cells $)$ than $3 \mathrm{D}$ cultures $\left(5 \times 10^{5}\right.$ cells $)$. After 5 days of culture, the point at which $3 \mathrm{D}$ cultures form solid spheroids and NP exposure began, total cell numbers in both cultures were approximately equal. Additionally, we normalized toxicology assay results with total DNA content.

We first investigated the cellular morphology change because it is an obvious initial sign of toxic effects. In $2 \mathrm{D}$ cell culture, HepG2 cells normally have a flat shape after spreading out on a well-plate while closely attaching to each other. However, their morphology dramatically changed after $12 \mathrm{~h}$ of CdTe exposure. A significant number of shrunk and rounded cells were observed with some partially detached from the well-plate (Figure 3A and B). Similarly CTAB-Au NPs induced substantial morphological change of cells in 2D culture, however there was negligible alteration of cellular structure in citrate-stabilized Au NP contact culture (Supporting Information Figure 5). No significant morphological alteration was noticed in 3D spheroid culture except only a slightly rugged spheroid surface in the CdTe and CTAB-Au NP-treated cultures (Figure 3C and D).

To distinguish more clearly between live and dead cells, we stained the cells with live/dead assay dyes and imaged them under a confocal microscope. In 2D culture, it was apparent that dead cells (red) morphed into a granular shape and fell away from the plate after losing their cell-cell and cellsubstrate interactions (Figure $3 \mathrm{E}$ and $\mathrm{F}$ ). Although the spheroid culture did not undergo a distinct morphological change, a few dead cells were observed on the surface of spheroids with a rugged exterior, as discussed above. There were noticeably fewer dead cells in the spheroid culture than the 2D culture (Figure $3 \mathrm{G}$ and $\mathrm{H}$ ).

We further characterized morphological change using a scanning electron microscope (SEM). In normal conditions, individual cells are hard to distinguish in both cultures because they form tight cell-cell junctions covered by a pervasively developed ECM layer (Figure 4A and D). After $12 \mathrm{~h}$ of CdTe NP exposure, dying or dead cells could be distinguished as they were separating from a live cell colony. This phenomenon was very obvious in 2D culture (Figure 4B). In spheroid culture, dead cells could be identified as protruding bulbs, but they did not separate from the spheroid (Figure 4E). It seemed that cells in a spheroid were tightly packed together and formed intensive junctions with adjoining cells. Thus, dead cells could stay in the spheroid despite losing their cell-cell interactions, and the overall spherical shape could be maintained.

To further examine the toxic effects of CdTe NPs, we extended the exposure time to $24 \mathrm{~h}$. As expected, longer treatment caused severe damage in $2 \mathrm{D}$ culture. A significant number of cells were dead and detached from the well-plate. Even cytoskeletons of dead cells were readily identified 

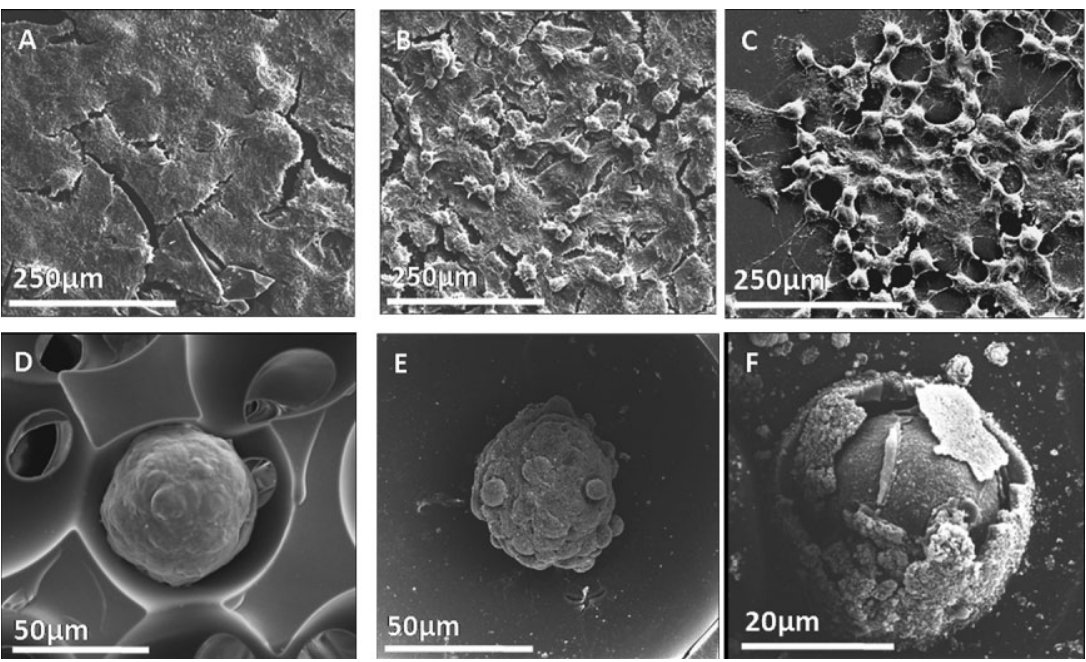

Figure 4. SEM images of 2D and 3D spheroid cultures before and after CdTe NP exposure. Typical morphology of A) 2D and D) 3D spheroid cultures after 5 days without CdTe NP exposure. The surface is very smooth due to tight cell-cell junctions and a well-developed ECM layer, making it hard to distinguish individual cells in both cultures. Representative morphology of B) 2D and E) 3D spheroid cultures after $12 \mathrm{~h}$ of CdTe NP treatment. Dying cells gradually lose their cell junctions. B) Shrunken cell bodies detached from the cell colony were easily recognized in 2D culture, while in 3D spheroid culture, dying or dead cells located on the surface were partially dissociated from the spheroid and appeared as protruded blobs (E). Morphological change of C) 2D and F) 3D spheroid cultures after $24 \mathrm{~h}$ of (dTe exposure. C) In $2 \mathrm{D}$ culture most of the cells were dead and a considerable number of cells were detached from the substrate. F) Although cells in spheroid culture were damaged, the multilayered structure buffered any toxic effects to inner cells. The intact inner cell mass was observed after partially detaching dead cell bodies on the surface of the spheroid by fixing the culture in formaldehyde for 1 week.

(Figure 4C). Similarly, in spheroid culture the surface roughness increased, corresponding to an increase in dead cell bodies. Individual dead cells were easily recognizable but they still remained on the spheroid. In order to observe the inner part of the spheroids, samples fixed in formaldehyde were left for 1 week to allow partial detachment of the dead cell bodies from the surface of the spheroid. Characterization of the spheroids revealed that the inner cell mass was preserved while the outer layer of cells was severely damaged (Figure 4F). CTAB-Au NPs showed similar results to $\mathrm{CdTe}$ NP-treated culture. Again no significant morphological change was observed for citrate-Au NPs.

Our morphological study results clearly indicate that the degree of toxicity of CdTe and CTAB-Au NPs to the spheroid culture is substantially lower when compared to the $2 \mathrm{D}$ culture. The most obvious reason for that is the diffusion of NPs into the spheroid. Densely packed cells in the spheroid are covered by a well-developed layer of ECM common for all tissues that reduces the penetration of toxicants. Hence, the inner layer of cells received less damage than cells in the outer layer. Also the dead cells on the outer layer of the spheroid potentially acted as a temporal protective barrier against toxic materials as they increased the thickness of the ECM.

\subsection{LDH and MTT Assays}

In the next step, the toxic effects of NPs were evaluated quantitatively utilizing lactate dehydrogenase (LDH) and methyl tetrazolium (MTT) assays. The LDH assay detects the amount of LDH that leaks out through the plasma membrane of damaged cells. This extracellular protein assay protocol was identical in both 2D and spheroid cultures. The MTT assay measures the amount of enzymatically reduced MTT by the mitochondria. Thus, cell lyses utilizing a detergent or lyses buffer were required. In the scaffold-based spheroid culture system, an additional spheroid and scaffold destruction step was needed to make a homogeneous cell lysate. As a result, we tore down and sonicated the ICC scaffolds. To keep the same assay conditions, 2D culture samples were also treated in the same manner. Both cultures were treated with CdTe NPs for $12 \mathrm{~h}$ and $\mathrm{Au}$ NPs for $24 \mathrm{~h}$ before running the assays.

As expected from the morphological study, the toxic effects of CdTe NPs were significantly reduced in spheroid cultures (Figure 5). Specifically, the results showed five times lower LDH leakage and two times more reduction of mitochondrial activity than in $2 \mathrm{D}$ cultures. The different sensitivity of the assay results was expected due to the nature of each assay and the different cell phenotypes. For example, the LDH assay is more sensitive to the number of cells at the solution interface capable of leaking their cytosolic contents into the media. All of the cells in $2 \mathrm{D}$ culture are exposed to the solution, while only a small portion of cells in spheroid culture make direct contact with the solution interface. The remaining cells are enclosed by the outer layer of cells. For this reason, it caused a larger gap between two cultures. In the MTT assay, however, cells are dissolved before analysis and, therefore, the cell phenotype is more closely related than diffusivity or the number of exposed surface cells. In the case of spheroid culture, one can consistently see a considerably reduced proliferation rate that causes the accumulation of a quiescent cell phenotype, which in turn gradually reduces cell metabolic activity. ${ }^{[16]}$ Since mitochondria produce about $90 \%$ of the adenosine triphosphate (ATP) required for cell survival, down-regulated mitochondrial activity in spheroid culture caused significantly reduced MTT activity compared to $2 \mathrm{D}$ culture despite the similar number of cells in both cultures. ${ }^{[39]}$ Therefore, the MTT assay results after CdTe NP exposure are required to be calibrated with control samples. The drop in MTT activity was almost two times higher in 2D culture than 3D spheroid culture (Figure 5B). Similarly we observed a significantly reduced toxic effect of $\mathrm{CTAB}-\mathrm{Au}$ NPs in spheroid culture. More specifically, LDH leakage and decreased mitochondrial activity was three times lower in spheroid culture than 2D flat culture. Citrate-Au NPs slightly reduced mitochondrial activity (5\%), however there was no substantial change of LDH leakage in both $2 \mathrm{D}$ and $3 \mathrm{D}$ cultures (Figure 6). 

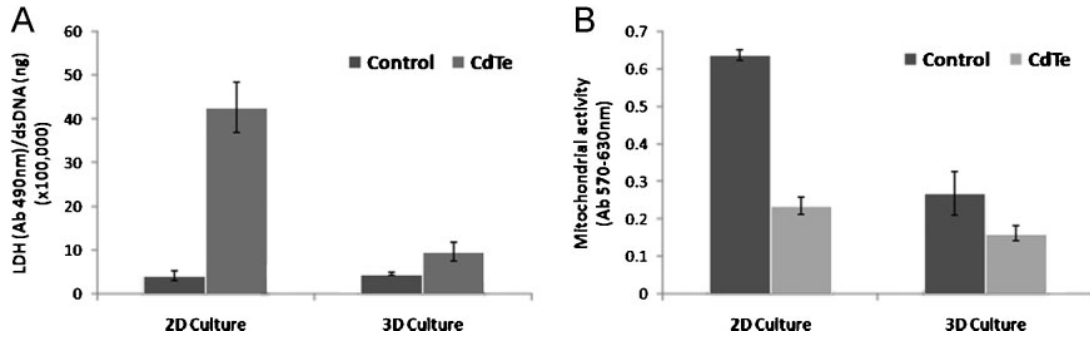

Figure 5. Quantitative comparison of toxicology assays between 2D and 3D spheroid culture after $12 \mathrm{~h}$ of CdTe NP exposure. A) Normalized LDH assay results. LDH activity before treatment was similar between the two cultures. However, after CdTe exposure, LDH leakage in $2 \mathrm{D}$ culture (1047\%) was almost five times higher than spheroid culture (212\%). B) MTT assay results. In control samples, MTT activity in the $2 \mathrm{D}$ culture was more than two times higher than the 3D culture due to down-regulated cellular metabolism. Upon exposure to CdTe NPs, the decrement of mitochondrial activity from the control group in the 2D culture (56\%) was approximately two times higher than the spheroid culture (31\%).

A

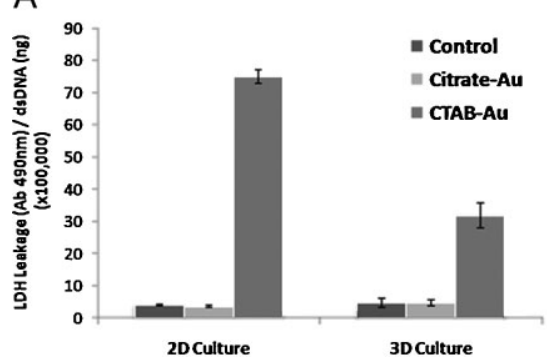

B

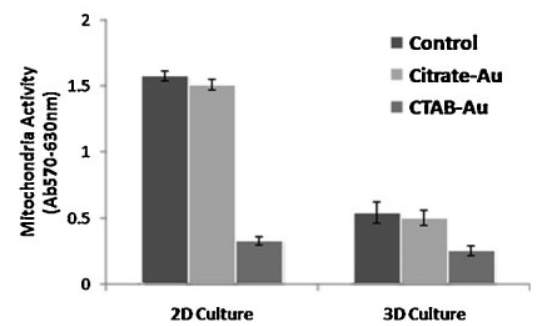

Figure 6. Quantitative comparison of toxicology assays between 2D and 3D spheroid culture after $24 \mathrm{~h}$ exposure to Au NPs having two different stabilizers. CTAB-Au NPs caused severe toxicity but citrate-Au NPs induced negligible toxic effects. CTAB-Au NP toxicity was significantly reduced when it was tested in 3D spheroid culture. A) Normalized LDH assay results demonstrate three times less LDH leakage of CTAB-Au NPs in 3D culture. B) MTT assay result shows that three times less reduction of mitochondrial activity of CTAB-Au NPs in 3D culture.

\subsection{Cell Death Mechanisms}

The MTT assay results reflect that reduced CdTe NP toxicity in spheroid culture is closely linked to a change of cell phenotype. With this in mind we hypothesized that CdTe NPinduced toxicity would cause different cell death mechanisms, that is, dying cells in spheroid culture would undergo more apoptosis but less necrosis than in 2D culture. Necrosis is a catastrophic cell death caused by acute cellular injury, resulting in the release of cytosolic proteins into the intercellular space. Apoptosis is a controlled, natural cell death mechanism. Compared to necrosis, the apoptotic process does not release intracellular constituents into the extracellular milieu but instead presents various signaling molecules such as caspase proteins. However, these signaling molecules are only temporarily presented before the cells undergo secondary necrosis, which is similar to necrotic cell death. ${ }^{[40]}$ Therefore, a time-dependent study is necessary to distinguish apoptosis from necrosis.

In order to test our hypothesis, the intensity of apoptotic (Caspase-3/7 assay) and necrotic (LDH assay) processes after $1,2,4,6,8,12$, and $24 \mathrm{~h}$ of CdTe NP exposure were measured (Figure 7). At these time points the culture medium was collected for the LDH assay, while the remaining cells on plates and scaffolds were further processed for the Caspase-3/7 assay. In both cultures, the apoptosis and necrosis values had similar trends yet they displayed substantial differences. In 2D cultures, the LDH assay value gradually increased until $8 \mathrm{~h}$ and then suddenly jumped, while in spheroid cultures it was slightly enhanced until $12 \mathrm{~h}$ followed by a moderate increase between 12 and $24 \mathrm{~h}$. Since a sudden increase of LDH leakage designates the point of prevalent necrosis or secondary necrosis at the end of apoptotic cell death, these data demonstrate that necrotic points of spheroid culture, either by necrosis or apoptosis, are much more retarded than 2D culture.

The caspase assay results more clearly showed phenotypic effects. In 2D culture, the caspase activity continuously decreased with different levels of retrenchment over time, gradually diminishing for $2 \mathrm{~h}$ before rapidly dropping. Please note that we intentionally used a relatively high concentration of unmodified CdTe NPs. On the other hand, in spheroid culture an initial jump in the caspase activity was first observed, followed by a gradual decrease. We also observed inherently higher caspase activity in 2D cultures than spheroid cultures similar to MTT results. Our data obviously indicate that cells in spheroid culture undergo more apoptotic processes than 2D culture due to the cellular phenotypic change.

Our results are well-correlated with previous animal testing results. ${ }^{[6,12-14,41]}$ For example, Zhang et al. reported that intravenous injection of CdTe NPs into rats did not cause

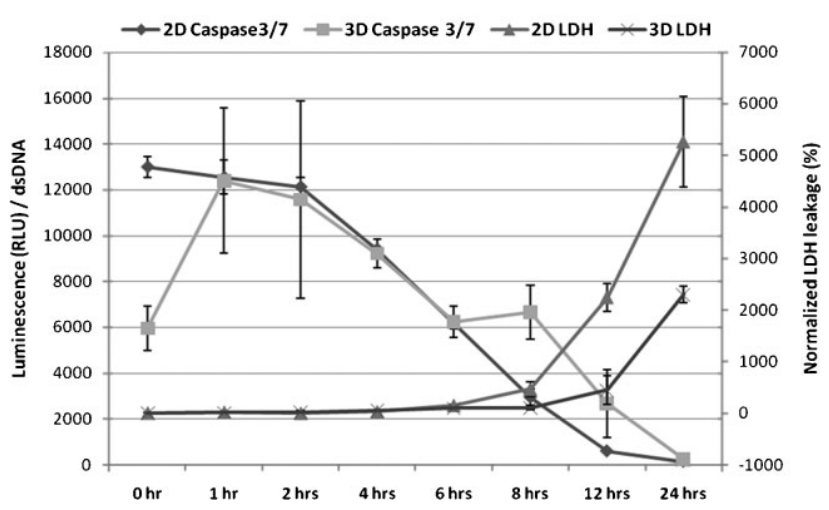

Figure 7. Kinetic studies of the cell death mechanism. Representative data of combined LDH and Caspase-3/7 assay results over a span of $24 \mathrm{~h}$, with measurements at eight different time points. The results were normalized with dsDNA quantification data. The LDH assay, the absorbance value on the right $y$-axis, was used as a necrosis marker. The Caspase- $3 / 7$ assay, the luminescence value on the left $y$-axis, was used as an apoptosis indicator ( $n=3$ at each time point, total $n=24$ in each type of culture, where $n$ is each independent experiment). 
any damage to major organs. Although locomotive activity was reduced shortly after dosing, it returned to normal within $24 \mathrm{h.}^{[11]}$ In fact, nanoscale materials undergo various physiological processes in the body, including circulating in the blood stream, accumulating in specific organs, cellular uptake, and renal excretion. ${ }^{[2-45]}$ It seems that such diverse and complex physiological processes cooperatively act to mitigate the toxic effects of NPs in vivo.

Similar to our findings, the reduced toxic effects of drug compounds in multicellular spheroid culture models were previously reported by other investigators. ${ }^{[46]}$ Also, there are several reports highlighting the unique opportunities of spheroid culture to an in vitro toxicity testing application. ${ }^{[47,48]}$ Nevertheless, it has not been widely accepted in the actual toxicity screening field because there are still technical barriers preventing current spheroid culture systems from being used in practical and industrial applications, such as standardization, reproducibility, high yields, and simple manipulation for experimental intervention and assay purposes. Hydrogel ICC scaffolds significantly improve all these issues. Highly controlled ICC structure and material resulted in excellent control and standardization of prepared liver tissue spheroids. A simple and versatile fabrication method allows the mass production of a diverse range of macro- and microscale ICC scaffolds. It can also be readily combined with currently utilized high-throughput screening (HTS) equipment. Additionally, the transparent hydrogel matrix enables the deep confocal 3D imaging of spheroids that is essential for high content analysis (HCA). ${ }^{[49,50]}$

In perspective, one can also envision other advantages of spheroid culture system. i) It enables long-term toxicity testing. Currently, all 2D culture-based toxicity testing is short term (less than a few days) due to continuous cell growth. However, longer toxicity testing is necessary to understand how toxic molecules affect cellular behavior not instantly but gradually, such as chronic exposure to toxic substances. The quiescent phenotype observed in spheroid culture is beneficial to treating cells for a longer period of time. ii) It can be used as a model system for understanding tissue-level healing processes after damage by toxic substances. As shown before, the interior spheroid was protected by an outer layer of cells, suggesting a capacity in spheroid culture for physiological repair, which is closer to real tissue biology. iii) Applying this system to tumor spheroids would be a very appropriate model system for testing the effectiveness of newly engineered NPs that are related to cancer treatments, such as tumor cell targeting and delivering therapeutics into solid tumors.

\section{Conclusions}

In this study, the 3D-cell-culture model for NP toxicity testing was introduced and great differences in comparison with common 2D cell cultures were demonstrated. Moreover, the data in $3 \mathrm{D}$ cell cultures obtained here correlate well with the data for animal tests, which show the future potential of this technique. Two important aspects of the 3D spheroid culture exemplify the differences with $2 \mathrm{D}$ cultures and the greater resemblance to in vivo tissue-like physiological responses: i) tissue-like mass transport due to dense tissuelike cell clusters and ECM layer and ii) cell phenotype changes due to intensified cell-cell interactions. Accurate prediction of potential toxicity of NPs in the body is an essential step to designate proper boundaries for their applications. The ICC scaffold-based spheroid culture system would be a valuable tool for undertaking this task as an initial testing platform, additionally producing physiologically relevant toxicological information. We also anticipate that it potentially can be applied to in vitro toxicity testing of new drug candidate compounds.

\section{Experimental Section}

Hydrogel ICC scaffold fabrication: The size of soda lime glass beads were controlled after multiple sieving steps to a diameter of $156.85 \pm 8.4 \mu \mathrm{m}$. CC templates $(D=6 \mathrm{~mm}, H=0.5-0.8 \mathrm{~mm}$, where $H$ is the height) were prepared following a previously described method. The CC was transferred to a glass vial having slightly larger dimension $(D=6.5 \mathrm{~mm})$. A prepared precursor solution composed of $30 \% \mathrm{w} / \mathrm{w}$ acrylamide, $5 \% \mathrm{w} / \mathrm{w} \mathrm{N}, \mathrm{N}$ methylenebisacrylamide, and $0.1 \% \mathrm{v} / \mathrm{v} N, N, N, N$-tetramethylethylenediamine in nitrogen-purged deionized water was infiltrated into the CC in a glass vial by centrifugation and polymerized upon addition of $1 \% \mathrm{w} / \mathrm{w}$ potassium peroxide solution. Upon hydrogel formation in the glass vial, the CC was separated from the bulk hydrogel and only the top part was thoroughly scratched using a razor blade. Subsequently, the glass beads were dissolved in $5 \%$ $\mathrm{v} / \mathrm{v}$ hydrogen fluoride solution for $24 \mathrm{~h}$. The ICC scaffolds were sequentially washed with acidic solution at $\mathrm{pH} 3.0$ for 1 day, phosphate-buffered saline (PBS) solution for 1 day, and deionized water for 2 days. Finally, the ICC scaffolds were freeze-dried and preserved in a dried state until use.

CdTe nanoparticle synthesis: $\mathrm{Cd}\left(\mathrm{ClO}_{4}\right)_{2} \cdot \mathrm{H}_{2} \mathrm{O}, \mathrm{HSCH}_{2} \mathrm{CH}\left(\mathrm{NH}_{2}\right)$ $\mathrm{CO}_{2} \mathrm{H}$ (L-cysteine), $\mathrm{H}_{2} \mathrm{SO}_{4}$, and $\left(\mathrm{C}_{8} \mathrm{H}_{16} \mathrm{CIN}\right)_{n}$ (poly(diallyldimethylammonium chloride), PDDA), were obtained from Sigma-Aldrich; $\mathrm{Al}_{2}$ Te was obtained from Cerac, Inc.; $\mathrm{NaOH}$ was obtained from Fluka; $\mathrm{CH}_{3} \mathrm{COCH}_{3}$ was obtained from Fisher; all chemicals were used without further purification; water was purified using $18 \mathrm{~m} \Omega$ deionized water (Barnstead E-pure System).

Atomic force microscopy (AFM) was performed with a Digital Instruments NanoScope Illa surface probe microscope. AFM specimens were prepared on a silicon wafer cleaned with acetone and subsequently soaked in $0.5 \%$ PDDA. AFM images were analyzed using NanoScope ${ }^{\circledR}$ III software tools. Fluorospectroscopic measurements were made using a Jobin Yvon Horiba FluoroMax-3.

L-Cysteine stabilized CdTe NPs were prepared according to the literature. ${ }^{[51,52]}$ Briefly, $2.35 \mathrm{mmol}$ of $\mathrm{Cd}\left(\mathrm{ClO}_{4}\right)_{2} \cdot \mathrm{H}_{2} \mathrm{O}$ and $5.7 \mathrm{mmol}$ of L-cysteine were dissolved in $125 \mathrm{~mL}$ of deionized water; the $\mathrm{pH}$ was rapidly adjusted to 11.2-11.4 using $1 \mathrm{~m} \mathrm{NaOH}$ and placed in a three-necked flask and deaerated with $\mathrm{N}_{2} . \mathrm{H}_{2}$ Te gas was introduced to this solution by the reaction of $0.46 \mathrm{mmol}$ $\mathrm{Al}_{2} \mathrm{Te}_{3}$ and $20 \mathrm{~mL}$ of $0.5 \mathrm{M} \mathrm{H}_{2} \mathrm{SO}_{4}$ in a separate three-necked flask. The solution was nitrogen-purged for an additional $30 \mathrm{~min}$, at which time the CdTe NP solution was refluxed to achieve the 
desired NP size. The resulting CdTe NPs were stabilized with a monolayer of L-cysteine, had an average diameter ranging from 2.4 to $6.0 \mathrm{~nm}$ (depending on the reflux time) with a corresponding photoluminescence ranging from 550 to $650 \mathrm{~nm}$, and a concentration on the order of $10^{-4}-10^{-6} \mathrm{~m}$, respectively.

Au nanoparticle synthesis: Au NPs having two different stabilizers were synthesized following the previously reported method. ${ }^{[53]}$ Briefly, a $20 \mathrm{~mL}$ of aqueous solution containing $2.5 \times 10^{-4} \mathrm{M} \mathrm{HAuCl}_{4}$ and $2.5 \times 10^{-4} \mathrm{M}$ trisodium citrate was mixed with $0.6 \mathrm{~mL}$ of ice-cold $0.1 \mathrm{M} \mathrm{NaBH}_{4}$ solution while stirring. In a few minutes, citrate-Au NPs were prepared with a diameter of $3.5 \pm 0.7 \mathrm{~nm}$. A $7.5 \mathrm{~mL}$ aqueous solution containing $2.5 \times 10^{-4} \mathrm{M}$ $\mathrm{HAuCl}_{4}$ and $0.08 \mathrm{M} \mathrm{CTAB}$ was mixed with $0.05 \mathrm{~mL}$ of $0.1 \mathrm{~m}$ of ascorbic acid solution. CTAB-Au NPs with a diameter of $5.5 \pm 0.6 \mathrm{~nm}$ were prepared by adding $2.5 \mathrm{~mL}$ of citrate-Au solution while stirring.

Cell culture and NP treatment: Rehydrated hydrogel ICC scaffolds in PBS solution were sterilized by immersion in $70 \%$ ethanol for 15 min under UV light. ICC scaffolds were then washed with PBS three times and transferred into a 48-well plate (Corning, NY). For 2D culture, a 12-well plate was used. Human hepatocellular carcinoma cell line HepG2 (HB-8065) was purchased from ATCC (Manassas, VA). Cell culture media was composed of William's E Medium supplemented with $10 \%$ fetal bovine serum (FBS) and $1 \%$ penicillin-streptomycin (Gibco). Cells were cultured in T-75 culture flasks at $37^{\circ} \mathrm{C}$ with $5 \% \mathrm{CO}_{2}$ until they reached the desired population. Cells were detached from the culture flask using $2.5 \%$ trypsin-ethylenediaminetetraacetic acid (EDTA) solution, and the concentration of the collected cell suspension was adjusted to $25 \times 10^{6}$ cells $\mathrm{mL}^{-1}$. $5 \times 10^{5}$ cells in $20 \mu \mathrm{L}$ were dropped on top of ICC scaffolds and then $1 \mathrm{~mL}$ of culture media was gently added. For $2 \mathrm{D}$ culture, $1 \times 10^{5}$ cells were seeded in each well of a 12 -well plate containing $1 \mathrm{~mL}$ of culture media. A half volume of media was changed daily for 5 days. On day 6 of culture, media was changed with $10 \mu \mathrm{g} \mathrm{mL}^{-1} \mathrm{CdTe}$ NP solution in William's E medium, and a control culture was maintained with only William's E medium. After incubation for 12 and $24 \mathrm{~h}$ (also some interval time points), CdTe exposure and control cultures were characterized.

Optical and confocal microscopes: Cell morphology was observed using an inverted microscope with $10 \times$ and $20 \times$ objectives (Nikon TS100) and a digital camera with imaging software (MicroPublisher, QImaging). Cell viability was observed using a Live/Dead Viability kit (Molecular Probes) and a Leica TCS SP2 confocal microscope (Leica Microsystems, Germany). CdTe exposure and control culture were incubated with $2 \mu \mathrm{m}$ calcein and $4 \mu \mathrm{M}$ ethidium homodimer- 1 for $40 \mathrm{~min}$ at $37^{\circ} \mathrm{C}$. Under a laser excitation of $488 \mathrm{~nm}$, live cells were imaged as green using a 510-540-nm emission filter, and dead cells stained by ethidium homodimer-1 were imaged as red using a 600-630-nm emission filter.

Scanning electron microscopy: SEM was used to characterize cellular morphology. Cells in sample scaffolds and on 2D glass slides were fixed overnight with $2.5 \%$ glutaraldehyde solution. The samples were then dehydrated through a series of ethanol solution concentrations of $50 \%, 70 \%, 90 \%, 95 \%$, and $100 \%$, and then freeze dried. Before imaging, completely dried samples were coated with Au (Desktop2, Venton Vacuum, Inc.). A FEI Nova
Nanolab (University of Michigan Electron Microbeam Analysis Laboratory) was used for SEM imaging.

LDH assay: LDH leakage from dead cells was analyzed using an LDH Assay kit (Promega, WI). A diluted supernatant of $50 \mu \mathrm{L}$ was mixed with $50 \mu \mathrm{L}$ of reagent and incubated for $30 \mathrm{~min}$ at room temperature. A stop solution of $50 \mu \mathrm{L}$ was then added, and absorbance at $490 \mathrm{~nm}$ was measured using a Synergy2 microplate reader (BioTek, VT).

$M T T$ assay: Mitochondria activity was quantified using an MTT Assay kit (ATCC, VA). The medium was aspirated and $500 \mu \mathrm{L}$ of fresh serum-free medium was added with $50 \mu \mathrm{L}$ of MTT reagent. After incubation for $3 \mathrm{~h}, 500 \mu \mathrm{L}$ of detergent solution was added and incubated for an additional $2 \mathrm{~h}$. The scaffolds and cell lyses solutions were transferred into $1.5 \mathrm{~mL}$ centrifuge tubes. Scaffolds were completely destroyed using forceps and further sonication. After centrifugation for $5 \mathrm{~min}$ at $1000 \mathrm{rpm}, 200 \mu \mathrm{L}$ of supernatant solution was collected and the absorbance was measured at $590 \mathrm{~nm}$ (sample) and $630 \mathrm{~nm}$ (reference). The difference in activity between normal and CdTe NP exposure cultures was used as a cytotoxicity indicator. All measurements were performed in triplicate, and six independent experiments were carried out.

Apoptosis assay: Caspase activity was measured using a Caspase-3/7 assay kit (Promega). After treatment of CdTe NPs, the culture media was completely removed for the LDH assay. The scaffolds were transferred into a $1.5-\mathrm{mL}$ centrifuge tube and $250 \mu \mathrm{L}$ of $1 \times$ cell lyses buffer solution (Promega) was added. The scaffolds were then crushed into small pieces with a microcentrifuge sample pestle and sonicated for 3-5 s. In 2D cultures, $250 \mu \mathrm{L}$ of $1 \times$ cell lyses buffer was added. Then $250 \mu \mathrm{L}$ of Caspase-3/7 assay reagent was added to each sample solution and incubated for $2 \mathrm{~h}$ at room temperature. To maintain the same sample preparation conditions, cell lysate in 2D cultures was transferred into a $1.5 \mathrm{~mL}$ centrifuge tube and briefly sonicated to make a homogeneous dispersion. Sample-containing centrifuge tubes were centrifuged for $5 \mathrm{~min}$ at $1000 \mathrm{rpm}$ to precipitate scaffolds or cell debris. $200 \mu \mathrm{L}$ of supernatant solution was collected in a white-wall luminescence plate. Luminescent intensity was measured using a microplate reader with a $10 \mathrm{~s}$ integration time.

\section{Acknowledgements}

The authors acknowledge the staff of the Electron Microscopy Analysis Laboratory (University of Michigan).

[1] P. L. Gartner, L. J. Hiatt, Color Text Book of Histology, Saunder, Philadelphia, PA 2001.

[2] A. Abbott, Nature 2003, 424, 870.

[3] J. Lee, M. J. Cuddihy, N. A. Kotov, Tissue Eng. B 2008, 14, 61.

[4] L. G. Griffith, M. A. Swartz, Nat. Rev. Mol. Cell. Biol. 2006, 7, 211.

[5] M. J. Bissell, A. Rizki, I. S. Mian, Curr. Opin. Cell. Biol. 2003, 15, 753.

[6] C. M. Sayes, K. L. Reed, D. B. Warheit, Toxicol. Sci. 2007, 97, 163.

[7] S. J. Cho, D. Maysinger, M. Jain, B. Roder, S. Hackbarth, F. M. Winnik, Langmuir 2007, 23, 1974. 
[8] T. R. Pisanic li, J. D. Blackwell, V. I. Shubayev, R. R. Fiñones, S. Jin, Biomaterials 2007, 28, 2572.

[9] A. Magrez, S. Kasas, V. Salicio, N. Pasquier, J. W. Seo, M. Celio, S. Catsicas, B. Schwaller, L. Forro, Nano Lett. 2006, 6, 1121.

[10] C. M. Sayes, J. D. Fortner, W. Guo, D. Lyon, A. M. Boyd, K. D. Ausman, Y. J. Tao, B. Sitharaman, L. J. Wilson, J. B. Hughes, J. L. West, V. L. Colvin, Nano Lett. 2004, 4, 1881.

[11] Y. Zhang, W. Chen, J. Zhang, J. Liu, G. Chen, C. Pope, J. Nanosci. Nanotechnol. 2007, 7, 497.

[12] C. M. Sayes, A. A. Marchione, K. L. Reed, D. B. Warheit, Nano Lett. 2007, 7, 2399.

[13] Z. Liu, C. Davis, W. Cai, L. He, X. Chen, H. Dai, Proc. Natl. Acad. Sci. USA 2008, 105, 1410.

[14] J. S. Kim, T.-J. Yoon, B. G. Kim, S. J. Park, H. W. Kim, K. H. Lee, S. B. Park, J.-K. Lee, M. H. Cho, Toxicol. Sci. 2006, 89, 338.

[15] K. M. Yamada, E. Cukierman, Cell 2007, 130, 601.

[16] R. M. Sutherland, Science 1988, 240, 177.

[17] K. Smalley, M. Lioni, M. Herlyn, In Vitro Cell. Dev. Biol.: Anim. 2006, 42, 242.

[18] K. Asano, N. Koide, T. Tsuji, J. Clin. Electron Microsc. 1989, 22, 243.

[19] M. Dvir-Ginzberg, I. Gamlieli-Bonshtein, R. Agbaria, S. Cohen, Tissue Eng. 2003, 9, 757.

[20] G. Oberdorster, E. Oberdorster, J. Oberdorster, Environ. Health Perspect. 2005, 113, 823.

[21] C. A. Poland, R. Duffin, I. Kinloch, A. Maynard, W. A. H. Wallace, A. Seaton, V. Stone, S. Brown, W. MacNee, K. Donaldson, Nat. Nanotechnol. 2008, 3, 423.

[22] R. Hardman, Environ. Health Perspect. 2006, 114, 165.

[23] V. L. Colvin, Nat. Biotechnol. 2003, 21, 1166.

[24] S. T. Stern, S. E. McNeil, Toxicol. Sci. 2008, 101, 4.

[25] A. Nel, T. Xia, L. Madler, N. Li, Science 2006, 311, 622.

[26] N. Lewinski, V. Colvin, R. Drezek, Small 2008, 4, 26.

[27] X. Gao, Y. Cui, R. M. Levenson, L. W. K. Chung, S. Nie, Nat. Biotechnol. 2004, 22, 969.

[28] J. Lee, S. Shanbhag, N. A. Kotov, J. Mater. Chem. 2006, 16, 3558.

[29] I. S. Vaithilingam, E. C. Stroude, W. McDonald, R. F. Maestro, J. Neuro-Oncol. 1991, 10, 203.

[30] N. A. Kotov, Y. Liu, S. Wang, C. Cumming, M. Eghtedari, G. Vargas, M. Motamedi, J. Nichols, J. Cortiella, Langmuir 2004, 20, 7887.

[31] G. Helmlinger, F. Yuan, M. Dellian, R. K. Jain, Nat. Med. 1997, 3, 177.

[32] R. Glicklis, J. C. Merchuk, S. Cohen, Biotechnol. Bioeng. 2004, 86, 672.

[33] J. Lovric, S. J. Cho, F. M. Winnik, D. Maysinger, Chem. Biol. 2005, $12,1227$.
[34] A. M. Derfus, W. C. W. Chan, S. N. Bhatia, Nano Lett. 2004, 4, 11.

[35] C. Kirchner, T. Liedl, S. Kudera, T. Pellegrino, A. MunozJavier, H. E. Gaub, S. Stolzle, N. Fertig, W. J. Parak, Nano Lett. 2005, 5, 331.

[36] A. Hoshino, K. Fujioka, T. Oku, M. Suga, Y. F. Sasaki, T. Ohta, M. Yasuhara, K. Suzuki, K. Yamamoto, Nano Lett. 2004, 4, 2163.

[37] C. J. Murphy, A. M. Gole, J. W. Stone, P. N. Sisco, A. M. Alkilany, E. C. Goldsmith, S. C. Baxter, Acc. Chem. Res. 2008, 41, 1721.

[38] Y. Pan, S. Neuss, A. Leifert, M. Fischler, F. Wen, U. Simon, G. Schmid, W. Brandau, W. Jahnen-Dechent, Small 2007, 3, 1941.

[39] P. F. James, J. Cell Physiol. 1998, 176, 138.

[40] A. Degterev, J. Yuan, Nat. Rev. Mol. Cell Biol. 2008, 9, 378.

[41] M. L. Schipper, N. Nakayama-Ratchford, C. R. Davis, N. W. S. Kam, P. Chu, Z. Liu, X. Sun, H. Dai, S. S. Gambhir, Nat. Nanotechnol. 2008, 3, 216.

[42] H. C. Fischer, L. Liu, K. S. Pang, W. C. Chan, Adv. Funct. Mater. 2006, 16, 1299.

[43] T. K. Jain, M. K. Reddy, M. A. Morales, D. L. Leslie-Pelecky, V. Labhasetwar, Mol. Pharm. 2008, 5, 316.

[44] R. Singh, D. Pantarotto, L. Lacerda, G. Pastorin, C. Klumpp, M. Prato, A. Bianco, K. Kostarelos, Proc. Natl. Acad. Sci. USA 2006, 103, 3357.

[45] H. S. Choi, W. Liu, P. Misra, E. Tanaka, J. P. Zimmer, B. Itty Ipe, M. G. Bawendi, J. V. Frangioni, Nat. Biotechnol. 2007, 25, 1165.

[46] T. M. Walker, P. C. Rhodes, C. Westmoreland, Toxicol. Vitro 2000, $14,475$.

[47] L. A. Kunz-Schughart, J. P. Freyer, F. Hofstaedter, R. Ebner, J. Biomol. Screen. 2004, 9, 273.

[48] R. Gebhardt, J. G. Hengstler, D. Muller, R. Glockner, P. Buenning, B. Laube, E. Schmelzer, M. Ullrich, D. Utesch, N. Hewitt, M. Ringel, B. R. Hilz, A. Bader, A. Langsch, T. Koose, H.-J. Burger, J. Maas, F. Oesch, Drug Metabol. Rev. 2003, 35, 145.

[49] P. O’Brien, W. Irwin, D. Diaz, E. Howard-Cofield, C. Krejsa, M. Slaughter, B. Gao, N. Kaludercic, A. Angeline, P. Bernardi, P. Brain, C. Hougham, Arch. Toxicol. 2006, 80, 580.

[50] E. Jan, S. J. Byrne, M. Cuddihy, A. M. Davies, Y. Volkov, Y. K. Gun'ko, N. A. Kotov, ACS Nano 2008, 5, 928.

[51] D. V. Talapin, A. L. Rogach, E. V. Shevchenko, A. Kornowski, M. Haase, H. Weller, J. Am. Chem. Soc. 2002, 124, 5782.

[52] N. Gaponik, D. V. Talapin, A. L. Rogach, K. Hoppe, E. V. Shevchenko, A. Kornowski, A. Eychmuller, H. Weller, J. Phys. Chem. B 2002, 106, 7177.

[53] N. R. Jana, L. Gearheart, C. J. Murphy, Langmuir 2001, 17, 6782.

Received: December 1, 2008 Published online: March 4, 2009 